\title{
Benjamin Taunay, Le Tourisme intérieur chinois
}

Collection “Espace et territoires", Presses universitaires de Rennes, 2011, 250 pages

\section{Ghislain Dubois}

\section{OpenEdition}

\section{Journals}

Édition électronique

URL : http://journals.openedition.org/tourisme/433

DOI : 10.4000/tourisme.433

ISSN : 2492-7503

\section{Éditeur}

Éditions touristiques européennes

\section{Édition imprimée}

Date de publication : 1 juin 2012

Pagination : 102-103

ISSN : 2109-5671

\section{Référence électronique}

Ghislain Dubois, «Benjamin Taunay, Le Tourisme intérieur chinois », Mondes du Tourisme [En ligne], 5 | 2012, mis en ligne le 30 septembre 2015, consulté le 22 septembre 2020. URL : http://

journals.openedition.org/tourisme/433; DOI : https://doi.org/10.4000/tourisme.433

Ce document a été généré automatiquement le 22 septembre 2020.

\section{cc)}

Mondes du tourisme est mis à disposition selon les termes de la licence Creative Commons Attribution

- Pas d'Utilisation Commerciale - Pas de Modification 4.0 International. 


\section{Lecture critique}

\section{Benjamin Taunay, Le Tourisme intérieur chinois}

Collection "Espace et territoires", Presses universitaires de Rennes, 2011, 250 pages

\section{Ghislain Dubois}

\section{RÉFÉRENCE}

Benjamin Taunay, Le Tourisme intérieur chinois, coll. “Espace et territoires”, Presses universitaires de Rennes, 2011.

1 L'ouvrage Le Tourisme intérieur chinois est intéressant d'abord par le choix de son sujet: quel acteur du tourisme ne souhaite pas aujourd'hui en savoir plus sur les Chinois? Mais aussi par le choix de s'occuper du tourisme intérieur, c'est-à-dire des pratiques des Chinois en Chine. Il faut sans cesse rappeler que le tourisme international (et marchand) n'est que la partie émergée du tourisme mondial. Les acteurs du tourisme, mais aussi parfois les scientifiques, en raison de la visibilité du tourisme international, s'appuient sur une vision totalement biaisée: un Parisien qui reste en France peut rapporter autant à l'économie qu'un Américain qui visite le pays; l'essentiel de la fréquentation européenne est du tourisme intérieur ou du tourisme intra-européen de proximité. Pourtant, chercheurs et acteurs restent fascinés par la quête des flux à longue distance. Ce qui peut se comprendre chez le directeur des Galeries Lafayette du boulevard Haussman, à Paris, est inexcusable à l'échelle d'un pays: une politique myope ne peut pas donner de bons résultats. Espérons que la Chine prenne un meilleur départ! En tous les cas, douze ans après l'instauration de la "semaine d'or" (en 1999) qui a généralisé l'accès des Chinois aux vacances, il n'était pas inutile de faire un point sur ce phénomène.

2 De bonne facture et bien illustré, l'ouvrage est très agréable à lire. Il prend une vraie distance avec les catégories statistiques du tourisme, proposant notamment une 
discussion sur le rôle des nationaux résidants à l'étranger dans le gonflement des chiffres impressionnants du tourisme récepteur chinois. Ici, l'analyse des dessous des statistiques révèle la même situation qu'en France : le tourisme chinois est d'abord un tourisme intérieur, ensuite un tourisme affinitaire de retour au pays, puis un tourisme asiatique "de proximité", enfin, mais plus marginalement, un tourisme international à longue distance. Ici, mais ce serait aller au-delà du but principal de l'ouvrage, l'auteur pourrait étendre la réflexion et contribuer à une nouvelle géographie du tourisme, plus conforme à la réalité des flux. S'il est compréhensible que le passage d'une frontière, qui a une efficacité administrative et une certaine réalité concrète pour distinguer des marchés reste un critère de premier ordre, il n'est pas acceptable de s'en limiter là : un Belge visitant Lille est un touriste international, contrairement à un New-Yorkais se rendant en Floride ou un Pékinois au Sichuan. Quid d'une redistribution des flux sur la base des distances, des différences de climat ou d'environnement? L'Europe ne serait plus en tête du tourisme mondial (ni la France), l'Amérique du Nord verrait son poids augmenté, et l'Asie apparaîtrait comme un acteur plus qu'émergent...

Par contre, on peut regretter que l'auteur, malgré une analyse poussée, fasse aussi facilement la confusion entre tourisme et agrément, jusqu'à intituler une partie "vrai et faux tourisme en Chine", négligeant ainsi le tourisme de visite à la famille et aux amis (pages 34, 43 et 77) ou en affirmant que les voyages professionnels ne sont pas du "vrai tourisme". En Chine comme ailleurs, le retour des migrants au pays ou les visites à une grand-mère urbaine ou campagnarde sont une réalité massive du tourisme, un facteur majeur expliquant à la fois les flux observés et les impacts économiques... Les négliger avec une telle erreur de définition, c'est retomber dans un travers similaire à celui évoqué plus haut.

4 Ensuite, l'ouvrage oscille agréablement entre analyse locale issue des expériences de son auteur dans la région de Guilin (province du Guizhou, sud de la Chine) et la fourniture d'informations sur l'ensemble du territoire chinois. Parmi les points intéressants ou particulièrement savoureux, on peut noter :

- l'affirmation d'une identité collective chinoise par le tourisme, mais aussi le renforcement de l'individu par rapport au collectif dans/grâce aux pratiques touristiques. Le tourisme confirme ici son rôle de révélateur et d'accompagnateur de dynamiques sociales qui le dépassent. Entre conformisme extrême et quête d'individualisme, le touriste chinois reste cependant un client paradoxal;

- la filiation entre pratiques anciennes et actuelles, le tourisme de la période communiste ayant suivi les pas du "tourisme lettré" des siècles précédents. On retrouve l'idée d'une résilience des schémas culturels présidant à la demande touristique, de même qu'une permanence des lieux touristiques au-delà des péripéties de l'histoire, aussi brutales soient-elles. Malgré un début de retour des touristes à la campagne, l'aversion à cet espace reste présente, la campagne étant associée par beaucoup à la révolution culturelle et à la rééducation des masses. Le pays connaît donc des phases de rejet et de redécouverte des traditions, mais au sein d'une certaine filiation des pratiques;

- la vision très paradoxale de l'authenticité et du patrimoine, les Chinois se satisfaisant tout à fait de reconstitutions, avec une "mise en scène qui mêle la tradition à la consommation" (p. 34), d'ailleurs souvent en milieu urbain - amener la campagne à la ville : très pragmatique... -, tout en exprimant une quête réelle d'authenticité. Au sein de cette recherche de modernité très compréhensible, le parc à thème est un lieu touristique marquant (2500 parcs en 2002 !). L'intérêt pour la nature est d'ailleurs caractérisé par la prédominance de la 
recherche d'une "signification imagée" (p. 234), les minorités ethniques y sont quelque peu rabaissées et le sentiment national glorifié ;

- le recours encore très limité à la voiture : les touristes de Guilin accèdent à la ville pour $36 \%$ en train, pour $48 \%$ en avion, et pour $3,3 \%$ seulement en voiture (p. 83).On peut imaginer que cela va changer...

- l'évocation d'une époque, si proche et si lointaine, où la Chine était fermée au monde : au plus fort de la révolution culturelle, le pays recevait, par l'intermédiaire de l'agence officielle, 4000 à 5000 touristes (483 en 1969 !). On croit rêver! Y a succédé un tourisme purement communiste, faisant coexister dans des stations balnéaires comme Beihai des cadres du parti et des unités de travail méritantes. Puis, mais seulement récemment, une démocratisation de l'accès aux vacances.

Enfin, fallait-il vraiment s'intéresser à la réalité d'un "modèle du tourisme chinois" dans la dernière partie de l'ouvrage pour rendre la recherche plus intéressante? Un modèle suppose des motivations, des logiques temporelles et spatiales, une organisation sociale qui génèrent un tourisme très différent. Or ici, à quelques nuances culturelles près, c'est au contraire une situation apparemment similaire à celle observée dans d'autres régions du monde : la réception de nouvelles pratiques et leur mélange avec des motifs de voyage plus traditionnels "pré-touristiques", la coexistence plus ou moins harmonieuse de ces pratiques, l'évolution de la structure spatiale pour accueillir ce nouveau flux... L'intérêt monographique du tourisme intérieur chinois est sans doute suffisamment intéressant sans qu'on en rajoute. L'idée selon laquelle "les formes et les lieux touristiques en Chine ne sont pas que la simple reproduction des modèles occidentaux" ne suffit pas à conclure à l'existence d'un "modèle". Encore un peu craintif et moutonnier, avide de consommation et grand utilisateur d'appareils photos, le touriste chinois d'aujourd'hui n'est-il pas simplement un "primo-touriste" ? Certaines évocations des pratiques contemporaines des Chinois rappellent en effet furieusement nos groupes de retraités et par là-même une vraie convergence des pratiques de tourisme, au-delà des époques et des lieux, même pour des cultures aussi éloignées que celles de l'Europe et de la Chine.

\section{AUTEURS}

\section{GHISLAIN DUBOIS}

Université de Versailles - Saint-Quentin-en-Yvelines 